\title{
Valutazione del danno epatico nel paziente nefropatico con infezione da HCV: quali sono le caratteristiche e i rischi dei test utili per l'inquadramento diagnostico
}

\author{
M.R. Brunetto \\ U.O. Gastroenterologia ed Epatologia \\ S.S.N. Azienda Ospedaliero-Universitaria, Pisa
}

$\mathbf{N}$ umerosi studi presenti in letteratura indicano che il danno epatico nel paziente con epatite cronica $\mathrm{C}$ progredisce più rapidamente dopo il trapianto di rene $(1,2)$.

$\mathrm{Ne}$ deriva che nel soggetto nefropatico candidato al trapianto di rene è necessario giungere, se possibile, all'eradicazione dell'infezione prima del trapianto o alternativamente procedere a una accurata stadiazione dell'epatopatia per individuare i soggetti che rischiano una rapida evoluzione dell'epatopatia nel post-trapianto.

Per l'inquadramento del paziente con infezione da HCV è necessario:

a. caratterizzare il profilo virologico (definire la durata dell'infezione, genotipo e livelli viremici);

b. procedere a una prima stadiazione non invasiva dell'epatopatia volta a definire la presenza/assenza di cirrosi con valutazione clinica, strumentale e bioumorale;

c. ricercare i co-fattori di danno epa- tico (alcol, altri virus, autoimmunità, dismetabolismo marziale, glico-lipido-sindrome dismetabolica);

d. nei soggetti senza segni bioumorali e/o strumentali di cirrosi alla valutazione di primo livello, occorre attuare un ulteriore approfondimento diagnostico volto alla definizione dello stadio della malattia; e. valutare la presenza e l'entità dell'ipertensione portale nei soggetti con evidenza di cirrosi alla valutazione di primo livello.

Gli accertamenti su indicati prevedono l'effettuazione di: 1) test di laboratorio (Tab. I);2) indagini strumentali non invasive; 3) indagini strumentali invasive.

\section{TABELLA I}

\section{Test di laboratorio:}

- Biochimica-clinica $\rightarrow$ attività biochimica-funzionalità epatica (dosaggio dei livelli di transaminasi, GGT, Aph, albumina, bil tot e frazionata, INR, CHE, protidogramma, dosaggio Ig, emocromo)

Co-fattori di danno (ferro, ferritina, transferrina, colesterolo, trigliceridi, glicemia, autoanticorpi)

- virologici $\rightarrow$ co-fattori (anti-HBc) caratterizzazione del profilo dell'infezione (genotipo, HCV- RNA quantitativo) 


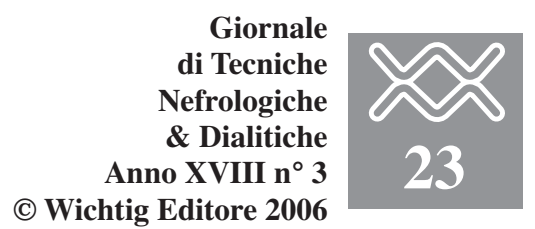

Indagini strumentali non invasive

ECT epato-splenica/eventuale doppler sistema portale $\rightarrow$ nella Tabella II sono riportati i dati relativi alla sensibilità, specificità, accuratezza diagnostica, potere predittivo positivo (PPV) e valore predittivo negativo (NPV) dell'ecografia nell'identificazione della cirrosi epatica. Il limite principale dell'ecografia deriva dalla notevole operatore-dipendenza della sua validità diagnostica $(3-8)$.

\section{Indagini invasive}

EGDScopia $\rightarrow$ per valutare la presenza di segni di ipertensione portale.

Agobiopsia epatica $\rightarrow$ l'esame microscopico del tessuto epatico permette di caratterizzare il danno epatico con l'identificazione delle lesioni elementari (distretti colpiti, presenza e tipo di infiltrato infiammatorio), la valutazione dell'entità del processo infiammatorio e della fibrosi e lo studio dell'architettura epatica.

Caratteristiche tecniche: la biopsia epatica viene effettuata, in genere, in regime di day hospital. Dal punto di vista tecnico, può essere effettuata senza o con controllo ecografico, in quest'ultimo caso può essere eco-assistita (individuazione del punto per l'effettuazione della biopsia con l'ecografia, ma effettuazione della biopsia a mano libera) o eco-guidata (individuazione del punto ed effettuazione della biopsia con guida ecografica).

Gli aghi che vengono utilizzati per la biopsia epatica possono essere ad aspi- razione (tipo Menghini), trancianti (tipo Tru-cut) o semi-automatici. Il calibro degli aghi varia da 1 (microistologia) a $1.6 \mathrm{~mm}$.

Nella Tabella III sono riportate le controindicazioni assolute e relative all'effettuazione della biopsia epatica (9). Una tecnica alternativa alla biopsia epatica percutanea è la biopsia transgiugulare, che può essere utilizzata se è presente ascite o importante alterazione della coagulazione. Con tale tecnica, tuttavia, si ottiene un frustolo spesso frammentato e più piccolo rispetto a quello ottenuto con la biopsia percutanea $(10-11)$.

\section{Criticità}

a) Morbilità e mortalità associate alla manovra: circa il $25 \%$ dei pazienti sot-

\section{TABELLA II}

\begin{tabular}{lcccccc}
\hline & $\begin{array}{c}\text { Gaiani } \\
\mathbf{1 9 9 7}\end{array}$ & $\begin{array}{c}\text { Zheng } \\
\mathbf{2 0 0 3}\end{array}$ & $\begin{array}{c}\text { Macias } \\
\mathbf{2 0 0 3}\end{array}$ & $\begin{array}{c}\text { Aube } \\
\mathbf{2 0 0 4}\end{array}$ & $\begin{array}{c}\text { Hung } \\
\mathbf{2 0 0 3}\end{array}$ & $\begin{array}{c}\text { Colli } \\
\text { Radiology }\end{array}$ \\
\hline Sensibilità (\%) & 82.2 & 62.5 & 80 & & 82.4 & \\
Specificità (\%) & 79.9 & 86.6 & 92 & & 70.7 & 95 \\
VPP (\%) & 54.4 & & & & 60.9 & 86 \\
VPN (\%) & 93.4 & & & & 87.8 & \\
Accuratezza diagnostica (\%) & 80.4 & 80.7 & 89 & $89-92^{*}$ & 74.8 & \\
\hline
\end{tabular}

\section{TABELLA III}

\section{Controindicazioni all'effettuazione della biopsia epatica}

\section{Assolute}

Paziente non collaborante

Anamnesi positiva per sanguinamenti inspiegati

Tendenza al sanguinamento (PT $\geq 3-5 \mathrm{sec}$ del controllo; $P L T S<50000 / \mathrm{mm}^{3}$; tempo di sanguinamento allungato $\geq 10 \mathrm{~min}$; uso di FANS nei precedenti $7-10 \mathrm{gg}$ )

Sangue per trasfusione non disponibile

Presenza o sospetto emangioma o altra neoplasia vascolare

Impossibilità di reperire sito adeguato per l'effettuazione della biopsia

Sospetta cisti da echinococco

\section{Relative}

Obesità patologica

Ascite

Emofilia

Infezione della cavità pleurica destra o al di sotto dell'emidiaframma $\mathrm{dx}$ 
toposti a biopsia lamenta dolore all'ipocondrio o alla spalla destri, occasionalmente il dolore induce una reazione vaso-vagale e in meno del $10 \%$ dei casi richiede l'utilizzo di analgesici. Piccoli ematomi intraepatici o sottocapsulari possono essere riscontrati all'ecografia di controllo in soggetti asintomatici.

Si stima che 1-3\% dei soggetti sottoposti a biopsia necessiti di ospedalizzazione per lo sviluppo di una complicanza, in genere autolimitantesi.

Il sanguinamento, costituisce la complicanza di maggiore rilevanza clinica: l'entità dello stesso può essere variabile, da minima a severa, tale da richiedere anche provvedimenti chirurgici. Va ricordato come segni di anemizzazione (tachicardia, ipotensione e riduzione dell'ematocrito) possano verificarsi anche a seguito della formazione di grossi ematomi intraepatici, che in genere devono essere sorvegliati clinicamente, senza approcci di tipo invasivo.

Più rara è l'emobilia, che può comparire a distanza di qualche giorno, manifestandosi con sanguinamento gastrointestinale, dolore biliare e ittero.

Le cause più frequenti di sanguinamento sono: movimenti inconsulti del paziente in corso di effettuazione della procedura; inclinazione dell'ago non perfettamente perpendicolare alla superficie epatica al momento dell'ingresso nel fegato; utilizzo di aghi tipo Tru-cut; puntura di un ramo della vena porta o dell'arteria epatica; più di 3 passaggi con l'ago; età avanzata del paziente, presenza di cirrosi; concomitanti patologie ematologiche o in pazienti in emodialisi.

Altre complicanze osservate a seguito dell'effettuazione di agobiopsia epatica sono: ascite biliare, pleurite biliare, peritonite biliare, pneumotorace, emotorace, enfisema sottocutaneo, pneumoperitoneo, pneumoscroto, ascesso sottofrenico, pancreatite secondaria a emobilia.

La mortalità viene stimata da 1 a 10 12000 , considerando anche le biopsie effettuate in soggetti sottoposti a biopsia per la diagnosi di lesioni maligne, condizione a maggior rischio di sanguinamento (9). b) Accuratezza diagnostica nella definizione del danno epatico: negli ultimi anni numerosi lavori hanno valutato il ruolo della biopsia epatica nella gestione del paziente affetto da epatite cronica C (12-19). In particolare, alcuni studi hanno avuto quale obiettivo primario la valutazione di quanto la biopsia epatica sia rappresentativa del danno epatico (in termini di necro-infiammazione e/o fibrosi) (16-18).

I lavori di Colloredo e Bedossa, che hanno cercato di definire le caratteristiche minime richieste al frustolo per garantire la consistenza del giudizio anatomo-patologico (possibilità di definire grading e staging) e hanno dimostrato come la biopsia dovrebbe avere una lunghezza di $2-2.5 \mathrm{~cm}$, un diametro di $1.4 \mathrm{~mm}$ e contenere almeno 11 spazi portali completi $(17,18)$.

Anche quando queste condizioni sono garantite, la possibilità di una disomogenea distribuzione del danno non può escludere in modo assoluto la persistenza di un certo errore di quantizzazione della fibrosi (16).

Inoltre, l'utilizzo di score (sistemi di semi-quantitativi, che utilizzano grandezze discontinue) limita la corretta stima della fibrosi, che al contrario è un fenomeno biologico continuo.

Infine, il giudizio diagnostico è condizionato dalla variabilità intra-, ma soprattutto interosservatore: bassa per quanto riguarda l'identificazione della cirrosi, moderata per la definizione degli stadi iniziali di fibrosi, alta per la definizione del grading (19).

Indicazione all'effettuazione della biopsia epatica: la biopsia epatica dovrebbe essere effettuata solo quando il suo apporto di conoscenza contribuisce al processo decisionale del medico.

Con questa premessa, l'indicazione alla biopsia epatica è presente:

a) ogniqualvolta, anche in presenza di cirrosi, sia necessario giungere a una precisa caratterizzazione del danno (ad esempio, ricerca di co-fattori), che potrebbero modificare la gestione del paziente;

b) nei soggetti senza evidenza di cirrosi alle indagini non invasive e per i quali sia ritenuta opportuna la stadiazione della fibrosi ai fini della de- cisione terapeutica;

c) nei pazienti per i quali sia ritenuto opportuno un monitoraggio della progressione di malattia

Nel caso del paziente nefropatico dovrà essere valutato attentamente il costo/beneficio della biopsia in considerazione dell'aumentato rischio di sanguinamento.

\section{Metodiche non invasive per la stadiazione della fibrosi}

Negli ultimi anni sono stati testati numerosi marcatori indiretti (Tab. V, 20) e diretti (Tab. IV, 21-23) per la definizione della fibrosi. Dai dati a ora disponibili è evidente che tali metodiche necessitano di una ulteriore fase di validazione clinica (applicazione su casistiche più ampie e diverse con confronto dei diversi marcatori e indici) prima che il loro utilizzo possa essere raccomandato nell'attività clinica routinaria.

Recentemente è stata proposta una nuova tecnica in grado di dare indicazioni circa la fibrosi epatica grazie alla misura dell'elastanza epatica (24-26). L'elastometria epatica è una metodica di recente introduzione che permette, attraverso la misurazione dell'elasticità del tessuto epatico, di valutare in modo non invasivo la fibrosi epatica.

La misura viene effettuata grazie a uno strumento Fibroscan ${ }^{\circledR}$ (EchoSens, Parigi, Francia), un device non invasivo che utilizza una sonda a ultrasuoni posizionata su un vibratore e connessa a un sistema elettronico. Il vibratore trasmette delle vibrazioni di media ampiezza e bassa frequenza che generano un'onda elastica $(50 \mathrm{~Hz})$, che si propaga nel fegato. La contemporanea acquisizione in eco-pulsata permette di seguire la propagazione dell'onda elastica e di misurarne la velocità, che è direttamente correlata alla rigidità (stiffness) del fegato. Le misure vengono effettuate a livello del lobo $\mathrm{dx}$, posizionando la sonda a livello degli spazi intercostali con paziente sdraiato, sotto controllo ecografico ai fini di evitare strutture vascolari maggiori. La valutazione si base su almeno 10 misurazioni validate, con un success rate (rapporto fra il numero di misurazioni validate e complessiva- 
TABELLA IV - MARCATORI DIRETTI DEL RIMANEGGIAMENTO DELLA MATRICE EXTRACELLULARE E DI FIBROSI

\author{
Marcatori della produzione di matrice \\ Procollageno I C terminale \\ Procollageno III $\mathrm{N}$ terminale \\ Tenascin \\ Inibitore tissutale della metalloproteinasi TIMP \\ TGF-beta \\ Marcatori di rimozione della matrice \\ Procollageno IV C peptide \\ Procollageno IV N peptide \\ Collageno IV \\ Undulina \\ Metalloproteinasi MMP \\ Desmosina urinaria \\ Dubbi \\ Acido ialuronico \\ Laminino \\ YKL-40
}

\section{TABELLA V - MARCATORI INDIRETTI DI FIBROSI EPATICA}

$\begin{array}{ll}\text { PGA } & \text { PT index, GGT e apolipoproteina A1 } \\ \text { PGAA test } & \text { PT index, GGT, apolipoproteina A1 e alfa 2-macroglobulina } \\ \text { Fibrotest } & \alpha-2 \text { macroglobulina, } \alpha \text { - 2 globuline, } \gamma \text {-globuline, } \\ & \begin{array}{l}\text { apolipoproteina A1, } \\ \text { GGT, bil tot }\end{array} \\ \text { Forns fibrosis index } & \text { Età, PLTS, GGT, colesterolo } \\ \text { APRI } & \text { AST/PLTS }\end{array}$

mente effettuate) $\geq 60 \%$ e un inter-quartile range $<20 \%$. La stiffness è espressa in kilopascals $(\mathrm{kPa})$ come il valore mediano di tutte le misure.

Abbiamo recentemente valutato la performance diagnostica dell'elastometria epatica in 228 pazienti affetti da epatite cronica $\mathrm{B}$ e/o $\mathrm{C}$, dimostrando che valori di Fibroscan $\geq 8.3 \mathrm{kPa}$ e $\geq 14 \mathrm{kPa}$ hanno un'accuratezza diagnostica dell' $87.3 \%$ e dell' $88.2 \%$ nell'identificazione dei soggetti con fibrosi epatica $\geq \mathrm{F} 2$ o cirrosi rispettivamente. Oltre ad aver confermato i dati pubblicati circa un'ottima correlazione fra valori dell'elastometria epatica e fibrosi, abbiamo inoltre dimostrato come fluttuazioni maggiori dell'attività biochimica di malattia possano influenzare il valore dell'elastometria epatica. Tale evidenza è in accordo con l'ipotesi che oltre alla fibrosi

\section{BIBLIOGRAFIA}

1. Gane E, et al Management of chronic viral hepatitis before and after renal transplantation Transplantation 2002; 74(4): 427-37.

2. Fabrizi F, et al. Treatment of hepatitis $\mathrm{C}$ in potential kidney and heart transplant patients Clin Liver Dis 2005; 9(3): 487-503.

3. Gaiani S, et al. What is the criterion for differentiating chronic hepatitis from compensated cirrhosis? A prospective study comparing ultrasonography and percutaneous liver biopsy. J Hepatol 1997; 27(6): 979-85.

4.

Zheng RQ, et al. Liver fibrosis in chronic viral hepatitis: an ultrasonographic study. World J Gastroenterol 2003; 9(11): 2484-9.

5. Macias Rodriguez MA, et al. Ultrasonography in patients with chronic liver disease: its usefulness in the diagnosis of cirrhosis. Rev Esp Enferm Dig 2003; 95(4): 258-64, 251-7.

6.

Aube C, et al. New Doppler ultrasound signs improve the non-invasive diagnosis of cirrhosis or severe liver fibrosis. Eur J Gastroenterol Hepatol 2004; 16(8):743-51.

7. Hung CH, et al. Correlation between ultrasonographic and pathologic diagnoses of hepatitis $\mathrm{B}$ and $\mathrm{C}$ virus-related cirrhosis. J Gastroenterol 2003; 38(2): 153-7.

dividuano l'elastometria epatica come una tecnica in grado di fornire informazioni utili all'inquadramento e stadiazione non invasiva dell'epatopatia. L'elastometria epatica potrebbe quindi diventare, dopo un'adeguata validazione clinica, un utile complemento diagnostico per l'inquadramento del paziente nefropatico candidato al trapianto epatico e potrebbe permettere di ridurre la necessità di effettuare la biopsia epatica, che nel soggetto nefropatico è gravata da un maggior rischio di sanguinamento.

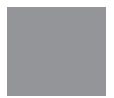

8. Colli A, et al. Severe liver fibrosis or cirrhosis: accuracy of US for detection-analysis of 300 cases. Radiology 2003; 227(1): 89-94. Epub 2003 Feb 19.

9. Bravo AA, Sheth SS, Chopra S. Liver Biopsy. N E J Med 2001, 344:7; 495-500.

10. Cholongitas E, et al. Transjugular liver biopsy: how good it is for accurate histological interpretation. Gut 2006.

11. Ishikawa T. Comparison of a new aspiration needle device and the QuickCore biopsy needle for tansjugular liver 


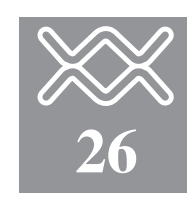

biopsy. World Gastroenterol 2006; 12(39): 6339-42.

12. Perrillo RP. The role of liver biopsy in hepatitis C. Hepatology 1997; 26(Suppl 1): S57-61.

13. Dienes HP, et al. Liver biopsy in hepatitis C. J Hepatol 1999; 31(Suppl 1): $43-6$.

14. Gebo KA, et al. Role of liver biopsy in management of chronic hepatitis C: a systematic review. Hepatology 2002; 36(Suppl 1): S161-72.

15. Dienstag JL. The role of liver biopsy in chronic hepatitis C. Hepatology 2002; 36(Suppl 1): S152-60.

16. Regev A, et al. Sampling error and intraobserver variation in liver biopsy in patients with chronic HCV infection. Am J Gastroenterol 2002; 97: 2164-8.

17. Colloredo G, et al. Impact of liver biopsy size on histological evaluation of chronic viral hepatitis: the smaller the sample, the milder the disease. J Hepatol 2003; 39: 239-44.

18. Bedossa P, et al. Sampling variability of liver fibrosis in chronic hepatitis C. Hepatology 2003; 38: 1449-57.

19. The METAVIR cooperative group Inter- and intra-observer variation in the assessment of liver biopsy of chronic hepatitis C. Hepatology 1994; 20; $1: 15-20$

20. Afdhal NH, Nunes D. Evaluation of liver fibrosis: a Concise review. Am J Gastroenterol 2004: 1160-74.

21. Myers RP, et al. Prediction of liver histological lesions with biochemical markers in patients with chronic hepatitis B. J Hepatol 2003; 39(2): 22230.

22. Poynard T, et al. Prospective analysis of discordant results between biochemical markers and biopsy in patients with chronic hepatitis C. Clin Chem 2004; 50(8): 1344-55. Epub 2004 Jun 10.

23. Callewaert N, et al. Noninvasive diagnosis of liver cirrhosis using DNA sequencer-based total serum protein glycomics. Nat Med 2004; 10(4): 42934.

24. Castera L, et al. Prospective comparison of transient elastography, Fibrotest, PRI, and liver biopsy for the assessment of fibrosis in chronic hepatitis C. Gastroenterology 2005; 128(2): $343-50$

25. Sandrin L, et al. Transient elastography: a new noninvasive method for assessment of hepatic fibrosis. Ultrasound Med Biol 2003; 29(12): 170513.

26. Coco B, et al. Transient elastography, a new surrogate marker of liver fibrosis influenced by major changes of transaminases. J Vir Hep (in press). 